VoL. 51 (1995) [263-272]

\title{
BEST APPROXIMATION THEOREMS FOR COMPOSITES OF UPPER SEMICONTINUOUS MAPS
}

\author{
SeHIE PARK
}

\begin{abstract}
Let $(E, \tau)$ be a Hausdorff topological vector space and $(X, w)$ a weakly compact convex subset of $E$ with the relative weak topology $w$. Recently, there have appeared best approximation and fixed point theorems for convex-valued upper semicontinuous maps $F:(X, w) \rightarrow 2^{(E, \tau)}$ whenever $(E, \tau)$ is locally convex. In this paper, these results are extended to a very broad class of multifunctions containing composites of acyclic maps in a topological vector space having sufficiently many linear functionals. Moreover, we also obtain best approximation theorems for classes of multifunctions defined on approximatively compact convex subsets of locally convex Hausdorff topological vector spaces or closed convex subsets of Banach spaces with the Oshman property.
\end{abstract}

\section{INTRODUCTION}

There have appeared many generalisations and applications of the best approximation theorems due to Ky Fan [6]. Recently, Ding and Tan [5, Theorems 4-6] obtained another generalisations for convex-valued upper semicontinuous multifunctions defined on a weakly compact convex subset of a locally convex Hausdorff topological vector space. These new results extend well known theorems due to Browder [2], Fan [6], Reich $[16,17,18,19]$, Ha [8], Park $[11,12]$, and many others.

In this paper, we show that those results can be extended to a very broad class of multifunctions containing composites of acyclic maps in a topological vector sapce having sufficiently many linear functionals. Our arguments are based on recent results due to the author $[13,14]$, and our new results on best approximations in the present paper are stated for multifunctions whose domains and ranges may have different topologies.

Moreover, we also obtain best approximation theorems for classes of multifunctions defined on approximatively compact convex subsets of a locally convex Hausdorff topological vector space or closed convex subsets of a Banach space with the Oshman property.

Received 30th May, 1994

The author was partially supported by the BSRI Program, MOE in 1993 and was a Visiting Professor at Indiana University, Bloomington, when this work was completed.

Copyright Clearance Centre, Inc. Serial-fee code: 0004-9729/95 \$A2.00+0.00. 


\section{Preliminaries}

A multifunction or set-valued map (simply, map) $F: X \rightarrow 2^{Y}$ is a function with nonempty set-values $F x \subset Y$ for each $x \in X$. The set $\{(x, y): y \in F x\}$ is called either the graph of $F$ or, simply, $F$. So $(x, y) \in F$ if and only if $y \in F x$.

For topological spaces $X$ and $Y$, a map $F: X \rightarrow 2^{Y}$ is upper semicontinuous (u.s.c.) if, for each closed set $B \subset Y, F^{-1}(B)=\{x \in X: F x \cap B \neq \emptyset\}$ is closed in $X$; and compact if $F(X)=\bigcup\{F x: x \in X\}$ is contained in a compact subset of $Y$. A nonempty topological space is acyclic if all of its reduced Cech homology groups over the rationals vanish.

A convex space $C$ is a nonempty convex set with any topology that induces the Euclidean topology on the convex hulls of its finite subsets. Such convex hulls are called polytopes. See [9].

Given a class $\mathbb{X}$ of maps, $\mathbb{X}(X, Y)$ denotes the set of all maps $F: X \rightarrow 2^{Y}$ belonging to $\mathbb{X}$, and $\mathbb{X}_{c}$ the set of all finite composites of maps in $\mathbb{X}$.

A class $\mathfrak{A}$ of maps is one satisfying the following:

(i) $\mathfrak{A}$ contains the class $\mathbb{C}$ of (single-valued) continuous functions;

(ii) each $F \in \mathfrak{A}_{c}$ is u.s.c. and compact-valued; and

(iii) for any polytope $P$, each $F \in \mathfrak{A}_{c}(P, P)$ has a fixed point.

Examples of $\mathfrak{A}$ are $\mathbb{C}$, the Kakutani maps $\mathbb{K}$ (with convex values), the acyclic maps $\mathbf{V}$ (with acyclic values), the approachable maps $\mathbb{A}$ in topological vector spaces [3], admissible maps in the sense of Górniewicz [7], permissible maps in Dzedzej [4], and others. Moreover, we define

$F \in \mathfrak{A}_{c}^{\sigma}(X, Y) \Longleftrightarrow$ for any $\sigma$-compact subset $K$ of $X$, there is a $\Gamma \in \mathfrak{A}_{c}(K, Y)$ such that $\Gamma x \subset F x$ for each $x \in K$.

$F \in \mathfrak{A}_{c}^{\kappa}(X, Y) \Longleftrightarrow$ for any compact subset $K$ of $X$, there is a $\Gamma \in \mathfrak{A}_{c}(K, Y)$ such that $\Gamma x \subset F x$ for each $x \in K$.

A class $\mathfrak{A}_{c}^{\kappa}$ is said to be admissible. Note that $\mathfrak{A} \subset \mathfrak{A}_{\mathfrak{c}} \subset \mathfrak{A}_{c}^{\sigma} \subset \mathfrak{A}_{c}^{\kappa}$. Examples of $\mathfrak{A}_{c}^{\sigma}$ are $\mathbb{K}_{c}^{\sigma}$ due to Lassonde [10] and $\mathrm{V}_{c}^{\sigma}$ due to Park, Singh, and Watson [15]. Note that $\mathbb{K}_{c}^{\sigma}$ includes classes $\mathbb{K}, \mathbb{R}$, and $\mathbb{T}$ in $[\mathbf{1 0}]$.

Let $E=(E, \tau)$ be a topological vector space, $E^{*}$ its topological dual, and $S(E)=$ $S(E, \tau)$ the family of all continuous seminorms on $(E, \tau)$. Let $w$ denote the weak topology of $E$. We say that $E^{*}$ separates points of $E$ if for each $x \in E$ with $x \neq 0$, there exists a $\phi \in E^{*}$ such that $\phi(x) \neq 0$; that is, if $x \neq 0$, then $p(x)>0$ for some $p \in S(E, w) \subset S(E, \tau)$ by taking $p(x)=|\phi(x)|$ for all $x \in E$.

The following are due to the author :

Lemma 1. [13, Theorem 4] Let $X$ be a nonempty convex subset of a locally convex Hausdorff topological vector space $E$, and $F \in \mathfrak{A}_{c}^{\sigma}(X, X)$. If $F$ is compact, 
then $F$ has a fixed point.

Lemma 2. [14, Theorem 3] Let $X$ be a compact convex space, $Y$ a Hausdorff space, and $F \in \mathfrak{2}_{c}^{\kappa}(X, Y)$. Let $\phi: X \times Y \rightarrow \mathbb{R}$ be a continuous function such that for each $y \in Y, x \mapsto \phi(x, y)$ is quasiconvex on $X$. Then there exists an $\left(x_{0}, y_{0}\right) \in F$ such that

$$
\phi\left(x_{0}, y_{0}\right) \leqslant \phi\left(x, y_{0}\right) \quad \text { for all } \quad x \in X .
$$

Let $C$ be a nonempty subset of a Hausdorff topological vector space $E$ and $p \in$ $S(E)$. For each $y \in E$, define $d_{p}(y, C)=\inf \{p(y-x): x \in C\}$ and the set of best approximations to $y \in E$ from $C$ by $Q_{p}(y)=\left\{x \in C: p(y-x)=d_{p}(y, C)\right\}$. We use $d$ instead of $d_{p}$ if $p$ is a norm. The multifunction $Q_{p}$ thus defined is called the metric projection onto $C$ if $Q_{p}(y) \neq \emptyset$ for each $y \in E$.

In $(E, \tau)$, let $\mathrm{Bd}$, Int, and - denote the boundary, interior, and closure, respectively, with respect to $\tau$.

The inward and outward sets of $X \subset E$ at $x \in E, I_{X}(x)$ and $O_{X}(x)$, are defined as follows:

$$
\begin{aligned}
I_{X}(x) & =\{x+r(u-x): u \in X, r>0\}, \\
O_{X}(x) & =\{x+r(u-x): u \in X, r<0\} .
\end{aligned}
$$

For a topological space $X$, a real function $f: X \rightarrow \mathbb{R}$ is lower semicontinuous (1.s.c.) if $\{x \in X: f x>r\}$ is open for each $r \in \mathbb{R}$.

The following is well known:

Lemma 3. Let $X$ and $Y$ be topological spaces, $h: X \times Y \rightarrow \mathbb{R}$ l.s.c., and $F: X \rightarrow 2^{Y}$ a compact-valued u.s.c. multifunction. Then $x \mapsto \inf \{h(x, y): y \in F x\}$ is 1.s.c. on $X$.

\section{FOR COMPACT CONYEX SPACES}

We begin with the following best approximation theorem:

ThEOREM 1. Let $C$ be a compact convex space, $E$ a Hausdorff topological vector space containing $C$ as a subset, and $F \in \mathfrak{A}_{c}^{\kappa}(C, E)$. Let $p: E \rightarrow \mathbb{R}$ be a quasiconvex function such that

$$
(x, y) \mapsto p(x-y) \text { is continuous for }(x, y) \in C \times E .
$$

Then there exists an $\left(x_{0}, y_{0}\right) \in F$ such that

$$
p\left(x_{0}-y_{0}\right) \leqslant p\left(z-y_{0}\right) \text { for all } z \in C .
$$

Proof: Since $C$ is compact, we may assume that $F \in \mathfrak{A}_{c}(C, E)$. Let $\phi: C \times E \rightarrow$ $\mathbb{R}$ be defined by $\phi(x, y)=p(x-y)$ for $x \in C$ and $y \in E$. Then $\phi$ is continuous such that for each $y \in Y, x \mapsto \phi(x, y)$ is quasiconvex on $C$. Therefore, by Lemma 2 , the conclusion follows. 
EXAMPLES. 1. If $C$ has the relative topology with respect to $E$ and $p: E \rightarrow \mathbb{R}$ is a quasiconvex continuous function, then clearly $(x, y) \mapsto p(x-y)$ is continuous on $C \times E$. Moreover, for $x \in E$, let $C_{x}=\operatorname{co}(C \cup\{x\})$ and

$$
n_{C}(x ; p)=\left\{y \in E: p(x-y) \leqslant p(z-y) \quad \text { for all } \quad z \in C_{x}\right\} \text {. }
$$

Then the conclusion becomes as follows: there exists an $x_{0} \in C$ such that $F\left(x_{0}\right) \cap$ $n_{C}\left(x_{0} ; p\right) \neq \emptyset$. Therefore, Lassonde [9, Proposition 3.3.6] is a particular case of Theorem 1 for $\mathbb{K}$ and for locally convex Hausdorff topological vector spaces.

2. Let $(C, \tau)$ and $(C, w)$ denote the set $C$ with the relative topology and the relative weak topology, respectively. Let $p \in S(E, w)$. Then $(x, y) \mapsto p(x-y)$ is continuous on $(C, w) \times(E, \tau)$ and on $(C, \tau) \times(E, w)$.

TheOREM 2. Let $C$ be a compact convex space, $E$ a Hausdorff topological vector space containing $C$ as a subset, and $F \in \mathfrak{A}_{c}^{\kappa}(C, E)$. Then, for each $p \in S(E, w)$ satisfying (*), there exists an $\left(x_{0}, y_{0}\right) \in F$ satisfying

$$
p\left(x_{0}-y_{0}\right)=d_{p}\left(y_{0}, \bar{I}_{C}\left(x_{0}\right)\right) .
$$

Moreover, $x_{0} \in \mathrm{Bd} C$ whenever $p\left(x_{0}-y_{0}\right)>0$.

Proof: By Theorem 1, we have an $\left(x_{0}, y_{0}\right) \in F$ such that

$$
p\left(x_{0}-y_{0}\right) \leqslant p\left(y_{0}-z\right) \quad \text { for all } z \in C .
$$

This implies

$$
p\left(x_{0}-y_{0}\right) \leqslant p\left(y_{0}-z\right) \text { for all } z \in I_{C}\left(x_{0}\right) .
$$

In fact, for $z \in I_{C}\left(x_{0}\right) \backslash C$, there exist $u \in C$ and $r>1$ such that $z=x_{0}+r\left(u-x_{0}\right)$. Suppose that $p\left(x_{0}-y_{0}\right)>p\left(y_{0}-z\right)$. Since

$$
\frac{1}{r} z+\left(1-\frac{1}{r}\right) x_{0}=u \in C,
$$

we have

$$
p\left(y_{0}-u\right) \leqslant \frac{1}{r} p\left(y_{0}-z\right)+\left(1-\frac{1}{r}\right) p\left(x_{0}-y_{0}\right)<p\left(x_{0}-y_{0}\right),
$$

a contradiction. Moreover, since $p \in S(E, w) \subset S(E, \tau)$ and $p$ is continuous on $(E, \tau)$, we have

$$
p\left(x_{0}-y_{0}\right) \leqslant p\left(y_{0}-z\right) \quad \text { for all } z \in \bar{I}_{C}\left(x_{0}\right) .
$$

Since $x_{0} \in \bar{I}_{C}\left(x_{0}\right)$, this implies

$$
p\left(x_{0}-y_{0}\right)=d_{p}\left(y_{0}, \bar{I}_{C}\left(x_{0}\right)\right) .
$$

Further, if $x_{0} \in \operatorname{Int} C$, then $I_{C}\left(x_{0}\right)=E$. Since $y_{0} \in E$, this implies $d_{p}\left(y_{0}, \bar{I}_{C}\left(x_{0}\right)\right)=$ $p\left(x_{0}-y_{0}\right)=0$. This completes our proof. 
REMARKS. 1. Note that, in Theorem 2, the topologies of $C$ and $E$ are related only by (*). Therefore, it is sufficient to assume that

(i) as a convex space, $C$ has any topology finer than the relative weak topology with respect to $E$, and

(ii) $E$ has any topology finer than its weak topology.

2. As in our previous work [14], for $F \in \mathfrak{A}_{c}^{\kappa}(C, E)$, define $F^{\prime}: C \rightarrow 2^{E}$ by $F^{\prime} x=2 x-F x$ for $x \in C$. If $F^{\prime} \in \mathfrak{A}_{c}^{\kappa}(C, E)$, then there exists an $\left(x_{0}, y_{0}\right) \in F$ such that

$$
p\left(x_{0}-y_{0}\right)=d_{p}\left(y_{0}, \bar{O}_{C}\left(x_{0}\right)\right) \text {. }
$$

Note that if $F$ belongs to $\mathbb{K}$ or $\mathbb{V}$, so does $F^{\prime}$.

Particular Forms. (1) Sehgal, Singh, and Smithson [21, Corollary 1] is a particular case of Theorem 2 for a locally convex Hausdorff topological vector space $(E, \tau)$ and for a single-valued map $F=f \in \mathbb{C}(C, w),(E, \tau))$.

(2) For a single-valued map $f \in \mathbb{C}((C, \tau),(E, \tau))$, Theorem 2 improves Reich [17, Theorem 1.6] for locally convex Hausdorff topological vector spaces and Fan [6, Theorem 2] for normed vector spaces.

From Theorem 2 we deduce the following form of Park [14, Theorem 4] with a different proof.

THEOREM 3. Let $C$ be a compact convex space, $E$ a topological vector space containing $C$ as a subset, and $F \in \mathfrak{A}_{c}^{\kappa}(C, E)$. Suppose that $E^{*}$ separates points of $E$ and that for each $p \in S(E, w)$, the condition $(*)$ holds. Then we have either

(1) F has a fixed point; or

(2) there exist a $p \in S(E, w)$, an $x_{0} \in \mathrm{Bd} C$, and a $y_{0} \in F x_{0}$ satisfying

$$
0<p\left(x_{0}-y_{0}\right)=d_{p}\left(y_{0}, \bar{I}_{C}\left(x_{0}\right)\right)
$$

Proof: We may assume that $F \in \mathfrak{A}_{c}(C, E)$. By Theorem 2, for each $p \in S(E, w)$, there exists an $\left(x_{0}, y_{0}\right) \in F$ such that $p\left(x_{0}-y_{0}\right)=d_{p}\left(y_{0}, \bar{I}_{C}\left(x_{0}\right)\right)$. Suppose that (2) does not hold. Then, for each $p \in S(E, w)$, there exists an $(x, y) \in F$ such that $p(x-y)=0$; that is;

$$
F[p]=\left\{x \in C: d_{p}(x, F x)=0\right\} \neq 0
$$

By putting $X=C, Y=(E, \tau)$, and $h(x, y)=p(x-y)$ in Lemma 3, we know that $x \mapsto d_{p}(x, F x)$ is l.s.c. Therefore, $F[p]$ is closed in $C$. Moreover, for a finite subset $\left\{p_{1}, p_{2}, \cdots, p_{n}\right\}$ of $S(E, w)$, we have $p=\sum_{i=1}^{n} p_{i} \in S(E, w)$ and $F[p]=F\left[\sum_{i=1}^{n} p_{i}\right] \subset$ 
$\bigcap_{i=1}^{n} F\left[p_{i}\right]$. Therefore, $\{F[p]: p \in S(E, w)\}$ is a family of closed subsets of $C$ with the finite intersection property. Since $C$ is compact, there exists a $u \in \bigcap\{F[p]: p \in$ $S(E, w)\}$.

Suppose that $u \notin F u$. Then the origin 0 does not belong to the compact set $K=u-F u$ of $(E, \tau)$. Let $z \in K$. Since $E^{*}$ separates points of $(E, w)$, there exists a $\phi \in E^{*}=(E, w)^{*}$ such that $\phi(z) \neq 0$. By putting $p_{z}(x)=|\phi(x)|$ for $x \in E$, we know that $p_{z} \in S(E, w) \subset S(E, \tau)$ and $p_{z}(z)>0$. Since $p_{z} \in S(E, \tau)$ is continuous on $K$, there exists an open neighbourhood $U_{z}$ of $z$ in $K$ such that $p_{z}(y)>0$ for every $y \in U_{z}$. Let $\left\{U_{z_{1}}, \cdots, U_{z_{k}}\right\}$ be a finite subcover of the cover $\left\{U_{z}\right\}_{z \in K}$ of $K$ and let $p_{u}=\sum_{i=1}^{k} p_{z_{i}} \in S(E, w)$. Since $\left.p_{u}\right|_{K}$ is continuous, it attains its infimum on $K$. Since the infimum can not be zero, we have $d_{p_{u}}(u, F u)>0$. This contradicts $u \in \bigcap\{F[p]: p \in S(E, w)\} \neq \emptyset$. This completes our proof.

Remark. As for Theorem 2, if $F^{\prime} \in \mathfrak{A}_{c}^{\kappa}(C, E)$, then the inward set in the conclusion (2) of Theorem 3 can be replaced by the outward set.

Particular Forms. Theorem 3 extends Ding and Tan [5, Theorem 4], where $(E, \tau)$ is locally convex and $F \in \mathbb{K}((C, w),(E, \tau))$, which in turn extends earlier works of Fan [6, Theorem 1], Browder [2, Corollaries 1 and $\left.1^{\prime}\right]$, Reich [16, Lemma 1.6], Ha [8, Theorem 3], and Park [12, Theorem 3].

Theorem 4. Under the hypothesis of Theorem $3, F$ has a fixed point $x_{0} \in C$ if one of the following conditions holds:

For each $x \in \operatorname{Bd} C \backslash F x$,

(0) for each $y \in F x$ and each $p \in S(E, w), p(x-y)>0$ implies

$$
p(x-y)>d_{p}\left(y, \bar{I}_{C}(x)\right) \text {. }
$$

(i) for each $y \in F x$, there exists a number $\lambda$ (real or complex, depending on whether $E$ is real or complex) such that

$$
|\lambda|<1 \text { and } \lambda x+(1-\lambda) y \in \bar{I}_{C}(x) .
$$

(ii) $F x \subset \bar{I}_{C}(x)$.

(iii) for each $y \in F x$, there exists a number $\lambda$ (as in (i)) such that

$$
|\lambda|<1 \quad \text { and } \lambda x+(1-\lambda) y \in C .
$$

(iv) $F x \subset I F_{C}(x)=\{x+c(u-x): u \in C, \operatorname{Re} c>1 / 2\}$.

(v) $F x \subset C$.

(vi) $F(C) \subset C$. 
Proof: (0) Clear from Theorem 3.

(i) For any $p \in S(E, w)$ satisfying $p(x-y)>0$, put $z=\lambda x+(1-\lambda) y$ in (0). Then we have

$$
p(y-z)=p(\lambda y-\lambda x)=|\lambda| p(x-y)<p(x-y)
$$

since $|\lambda|<1$.

(ii) If $F x \subset \bar{I}_{C}(x)$, then for each $y \in F x$, we can choose $\lambda=0$ in (i).

(iii) Since $C \subset I_{C}(x)$, we clearly have (iii) $\Longrightarrow$ (i).

(iv) It is well-known that (iv) $\Longleftrightarrow$ (iii).

(v) If $F x \subset C$, then for each $y \in F x$, we can choose $\lambda=0$ in (iii).

(vi) Clearly, we have (vi) $\Longrightarrow$ (v).

REMARK. If $F^{\prime} \in \mathfrak{A}_{c}^{\kappa}(C, E)$, then the inward sets in (0), (i), (ii), and (iv) can be replaced by the corresponding outward sets.

Particular Forms. (1) Ding and Tan [5, Theorems 5 and 6] are Theorem 4(0) and (i), respectively, for a locally convex Hausdorff topological vector space $(E, \tau)$ and $F \in \mathbb{K}((C, w),(E, \tau))$, which in turn extend earlier works of Fan [6, Theorem 3], Reich [16, Theorem 1.7], [18, Theorem 3.1], [19, Theorem 2], Ha [8, Theorem 4], and Park $[12$, Theorem 4].

(2) Roux and Singh [20, Theorems 5 and 6] are particular cases of Theorem 4(iii) for $F=f \in \mathbb{C}((C, \tau),(E, w))$ and $F=f \in \mathbb{C}((C, w),(E, \tau))$.

(3) Arino, Gautier, and Penot [1, Theorem 1] is a particular form of Theorem 4(v) for a weakly sequentially continuous funciton $F=f:(C, w) \rightarrow(C, w)$, where $(E, \tau)$ is a metrisable locally convex Hausdorff topological vector space.

(4) For other well-known particular forms of Theorem 4, see Park [14].

Theorem 4(vi) can be restated as follows:

Corollary 1. Let $C$ be a compact convex space, $E=(E, \tau)$ a topological vector space on which $E^{*}$ separates points such that $E$ contains $C$ as a subset and, for each $p \in S(E, w),(x, y) \mapsto p(x-y)$ is continuous for $(x, y) \in C \times E$. Then any $F \in \mathfrak{A}_{c}^{\kappa}(C,(C, \tau))$ has a fixed point.

From Corollary 1, we have the following:

Corollary 2. Let $C$ be a compact convex subset of a topological vector space $E$ on which $E^{*}$ separates points. Then any $F \in \mathfrak{A}_{c}^{\kappa}(C, C)$ has a fixed point.

Note that, even for $\mathfrak{A}=\mathbb{K}$, Corollary 2 includes historically well-known fixed point theorems of Brouwer (1912), Schauder (1927, 1930), Tychonoff (1935), Kakutani (1941), Bohnenblust and Karlin (1950), Fan (1952, 1964), Glicksberg (1952), Granas and Liu (1986), and many others. See Park [14]. 


\section{For APPROXIMATIVELY COMPACT SETS AND OTHERS}

In this section, we consider best approximation theorems for the admissible maps defined on approximatively compact convex subsets of a locally convex Hausdorff topological vector space or closed convex subsets of a Banach space with the Oshman property. Consequently, we improve some results in [15].

In the sequel, we assume that the class $\mathfrak{A}$ contains $\mathbb{K}$.

Let $C$ be a subset of a Hausdorff topological vector space $E$ and $p \in S(E)$. Then $C$ is called approximatively compact (with respect to $p$ ) if, for each $y \in E$, every net $\left\{x_{\alpha}: \alpha \in \Lambda\right\}$ in $C$ such that $p\left(y-x_{\alpha}\right) \rightarrow d_{p}(y, C)$ has a subnet that converges to an element of $C$. It is well-known that the metric projection $Q_{p}: E \rightarrow 2^{C}$ belongs to $\mathbb{K}(E, C)$ whenever $C$ is approximatively $p$-compact and convex.

THEOREM 5. Let $C$ be a nonempty approximatively compact, convex subset of a locally convex Hausdorff topological vector space $E$, and $F \in \mathfrak{A}_{c}^{\sigma}(C, E)$. If $F$ is compact, then for each $p \in S(E)$, there exsits an $\left(x_{0}, y_{0}\right) \in F$ such that

$$
p\left(x_{0}-y_{0}\right)=d_{p}\left(y_{0}, \bar{I}_{C}\left(x_{0}\right)\right)
$$

Moreover, $x_{0} \in \mathrm{Bd} C$ whenever $p\left(x_{0}-y_{0}\right)>0$.

Proof: Since $Q_{p} \in \mathbb{K}(E, C)$, by assumption, we have $Q_{p} \in \mathfrak{A}(E, C)$. Since $F \in \mathfrak{A}_{c}^{\sigma}(C, E)$ and $\mathfrak{A}_{c}^{\sigma}$ is closed under composition (see [10, Proposition 2(1)], we have $Q_{p} F \in \mathfrak{A}_{c}^{\sigma}(C, C)$. Note that $F$ is compact and that $Q_{p}$ is a compact-valued u.s.c. map. Hence, $Q_{p} F$ is compact. Therefore, by Lemma $1, Q_{p} F$ has a fixed point $x_{0} \in\left(Q_{p} F\right) x_{0}$; that is, there exists a $y_{0} \in F x_{0}$ such that

$$
x_{0} \in Q_{p} y_{0}=\left\{x \in C: p\left(x-y_{0}\right)=d_{p}\left(y_{0}, C\right)\right\}
$$

Actually, this implies the conclusion as for Theorem 2.

Particular Forms. For $F \in \mathrm{V}_{c}^{\sigma}(C, E)$, Theorem 5 is due to Park, Singh, and Watson [15, Theorem 3], which includes Reich [18, Corollary 2.2].

From Theorem 5, we obtain the following fixed point theorem for normed vector spaces.

Corollary 3. Let $C$ be an approximatively compact convex subset of a normed vector space $E$, and $F \in \mathfrak{A}_{c}^{\sigma}(C, E)$ compact. Suppose that

(0) for each $x \in \operatorname{Bd} C \backslash F x$ and $y \in F x$, we have

$$
\|x-y\|>\|z-y\| \quad \text { for some } z \in \bar{I}_{C}(x)
$$


Then $F$ has a fixed point.

Proof: By Theorem 5, there exists an $\left(x_{0}, y_{0}\right) \in F$ such that $\left\|x_{0}-y_{0}\right\| \leqslant$ $\left\|z-y_{0}\right\|$ for all $z \in \bar{I}_{C}\left(x_{0}\right)$. If $x_{0} \neq y_{0}$, then $x_{0} \in \mathrm{Bd} C$ and (0) leads a contradiction. This completes our proof.

Particular Forms. For $F \in \mathbb{V}_{c}^{\sigma}(C, E)$, Corollary 3 reduces to [15, Corollary 2]. Instead of the condition (0), we can adopt any of (i)-(vi) in Theorem 4.

Remark. As for Theorems 2-4, if $F^{\prime} \in \mathfrak{A}_{c}^{\sigma}(C, E)$, then the inward sets in Theorem 5 and Corollary 3 can be replaced by the corresponding outward sets.

Recall that a reflexive Banach space has the Oshman property if the metric projection on every closed convex subset belongs to $\mathbb{K}$.

THEOREM 6. Let $C$ be a closed convex subset of a Banach space $E$ with the Oshman property, and $F \in \mathfrak{A}_{c}^{\kappa}(C, E)$. If $F$ is compact, then there exists an $\left(x_{0}, y_{0}\right) \in F$ such that

$$
\left\|x_{0}-y_{0}\right\|=d\left(y_{0}, \bar{I}_{C}\left(x_{0}\right)\right) .
$$

ProOF: Let $Q: E \rightarrow 2^{C}$ be the metric projection. Then $Q \in \mathbb{K}(E, C) \subset \mathfrak{A}(E, C)$ and $Q F \in \mathfrak{A}_{c}^{\kappa}(C, C)$. Note that $Q F$ is compact. Let $K=\operatorname{co} \overline{Q F(C)}$ be the compact convex set in $C$. Then $Q F \in \mathfrak{A}_{c}^{k}(K, K)$. By Corollary $2, Q F$ has a fixed point $x_{0} \in C$; that is, $x_{0} \in(Q F) x_{0}$. Hence there exists a $y_{0} \in F x_{0}$ satisfying $x_{0} \in Q y_{0}=\{x \in C$ : $\left.\left\|\boldsymbol{x}-y_{0}\right\|=d\left(y_{0}, C\right)\right\}$. This implies the conclusion as for Theorem 2 .

Particular Forms. For $F \in \mathbb{V}_{c}^{\sigma}(C, E)$, Theorem 6 is due to [15, Theorem 4], which includes Reich [18, Proposition 2.3].

Corollary 4. Let $C$ be a closed convex subset of a Banach space $E$ with the Oshman property, and $F \in \mathfrak{A}_{c}^{\kappa}(C, E)$ compact. Suppose that the condition ( 0$)$ of Corollary 3 holds. Then $F$ has a fixed point.

The proof is like that of Corollary 3.

Particular Forms. For $F \in \mathbb{V}_{c}^{\sigma}(C, E)$, Corollary 4 is due to [15, Corollary 3], which includes Reich [18, Proposition 3.2 and Theorem 3.3(b)], [19, Theorems 6 and 7].

\section{REFERENCES}

[1] O. Arino, S. Gautier, and J.P. Penot, 'A fixed point theorem for sequentially continuous mappings with application to ordinary differential equations', Funkcial. Ekvac. 27 (1984), 273-279.

[2] F.E. Browder, 'On a sharpened form of the Schauder fixed point theorem', Proc. Nat. Acad. Sci. U.S.A. 74 (1977), 4749-4751. 
[3] M. Ben-El-Mechaiekh and P. Deguire, 'Approximation of non-convex set-valued maps', C.R.Acad. Sci. Paris 312 (1991), 379-384.

[4] Z. Dzedzej, 'Fixed point index theory for a class of nonacyclic multivalued maps', Dissertationes Math. 253 (1985).

[5] X.P. Ding and K.-K. Tan, 'A set-valued generalization of Fan's best approximation theorem', Canad. J. Math. 44 (1992), 784-796.

[6] Ky Fan, 'Extensions of two fixed point theorems of F.E. Browder', Math. Z. 112 (1969), 234-240.

[7] L. Górniewicz, 'Homological methods in fixed point theory of multivalued maps', Dissertationes Math. 129 (1976).

[8] C.W. Ha, 'On a minimax inequality of Ky Fan', Proc. Amer. Math. Soc. 99 (1987), 680-682.

[9] M. Lassonde, 'On the use of KKM multifunctions in fixed point theory and related topics', J. Math. Anal. Appl. 97 (1983), 151-201.

[10] M Lassonde, 'Réduction du cas multivoque au cas univoque dans les problèmes de coincidence', in Fixed point theory and applications, (M.A. Théra and J.B. Baillon, Editors) (Longman Scientific and Technical, Essex, 1991), pp. 293-302.

[11] S. Park, 'Fixed point theorems on compact convex sets in topological vector spaces', Contemp. Math. 72 (1988), 183-191.

[12] S. Park, 'Fixed point theorems on compact convex sets in topological vector spaces, II', J. Korean Math. Soc. 26 (1989), 175-179.

[13] S. Park, 'Foundations of the KKM theory via coincidences of composites of upper semicontinuous maps', J. Korean Math. Soc. 31 (1994), 493-519.

[14] S. Park, 'Fixed point theory of multifunctions in topological vector spaces, II', J. Korean Math. Soc. 30 (1993), 413-431.

[15] S. Park, S.P. Singh and B. Watson, 'Some fixed point theorems for composites of acyclic maps', Proc. Amer. Math. Soc. 121 (1994), 1151-1158.

[16] S. Reich, 'Fixed points in locally convex spaces', Math. Z. 125 (1972), 17-31.

[17] S. Reich, 'On fixed point theorems obtained from existence theorems for differential equations', J. Math. Anal. Appl. 54 (1976), 26-36.

[18] S. Reich, 'Approximate selections, best approximations, fixed points, and invariant sets', J. Math. Anal. Appl. 62 (1978), 104-113.

[19] S. Reich, 'Fixed point theorems for set-valued mappings', J. Math. Anal. Appl. 69 (1979), 353-358.

[20] D. Roux and S.P. Singh, 'On a best approximation theorem', Jñänābha 19 (1989), 1-9.

[21] V.M. Sehgal, S.P. Singh and R.E. Smithson, 'Nearest points and some fixed point theorems for weakly compact sets', J. Math. Anal. Appl. 128 (1987), 108-111.

\footnotetext{
Department of Mathematics

Seoul National University

Seoul 151-742

Korea
} 